\title{
ON SOLUTIONS OF QUASILINEAR ELLIPTIC INEQUALITIES CONTAINING TERMS WITH LOWER-ORDER DERIVATIVES
}

\author{
ANDREJ A. KON'KOV
}

\section{INTRODUCTION}

Let $\Omega$ be an unbounded open subset of $\mathbb{R}^{n}, n \geq 2$. As in [5], by $W_{p, l o c}^{1}(\Omega)$ we mean the space of measurable functions that belong to $W_{p}^{1}\left(B_{r} \cap \Omega\right)$ for all real numbers $r>0$ satisfying the condition $B_{r} \cap \Omega \neq \emptyset$, where $B_{r}$ is the open ball in $\mathbb{R}^{n}$ of radius $r$ and center at zero. The space $L_{\infty, l o c}(\Omega)$ is defined in a similar way.

Consider the inequality

$$
\operatorname{div} A(x, D u)+b(x)|D u|^{p-1} \geq q(x) g(u) \quad \text { in } \Omega,
$$

where $D=\left(\partial / \partial x_{1}, \ldots, \partial / \partial x_{n}\right)$ is the gradient operator, $b \in L_{\infty, l o c}(\Omega)$, and $A$ : $\Omega \times \mathbb{R}^{n} \rightarrow \mathbb{R}^{n}$ is a measurable function such that

$$
C_{1}|\zeta|^{p} \leq \zeta A(x, \zeta), \quad|A(x, \zeta)| \leq C_{2}|\zeta|^{p-1}
$$

with some constants $C_{1}>0, C_{2}>0$, and $p>1$ for almost all $x \in \Omega$ and for all $\zeta \in \mathbb{R}^{n}$.

We assume that $S_{r} \cap \Omega \neq \emptyset$ for all $r>r_{0}$, where $r_{0}>0$ is some real number and $S_{r}$ is the sphere in $\mathbb{R}^{n}$ of radius $r$ and center at zero. Also let $q \in L_{\infty, l o c}(\Omega)$ and $g \in C([0, \infty))$ be non-negative functions and, moreover, $g(t)>0$ for all $t>0$. We denote

$$
\begin{gathered}
f_{\sigma}(r)=\frac{\operatorname{essinf}_{\Omega_{r / \sigma, \sigma r}}^{\sin }|b|}{1+r \operatorname{ess} \sup \mid b}, \quad r>r_{0}, \sigma>1, \\
q_{\sigma}(r)=\underset{\Omega_{r / \sigma, \sigma r}}{\operatorname{essinf} q, \quad} \Omega_{r / \sigma, \sigma r}
\end{gathered}
$$

and

$$
g_{\theta}(t)=\inf _{(t / \theta, \theta t)} g, \quad t>0, \theta>1,
$$

where $\Omega_{r_{1}, r_{2}}=\left\{x \in \Omega: r_{1}<|x|<r_{2}\right\}, 0 \leq r_{1}<r_{2} \leq \infty$.

A non-negative function $u \in W_{p, l o c}^{1}(\Omega) \cap L_{\infty, l o c}(\Omega)$ is called a solution of inequality (1.1) if the map $x \mapsto A(x, D u)$ is measurable and

$$
-\int_{\Omega} A(x, D u) D \varphi d x+\int_{\Omega} b(x)|D u|^{p-1} \varphi d x \geq \int_{\Omega} q(x) g(u) \varphi d x
$$

for any non-negative function $\varphi \in C_{0}^{\infty}(\Omega)$. As is customary, the condition

$$
\left.u\right|_{\partial \Omega}=0
$$

means that $\psi u \in \stackrel{\circ}{W}_{p}^{1}(\Omega)$ for any $\psi \in C_{0}^{\infty}\left(\mathbb{R}^{n}\right)$. If $\Omega=\mathbb{R}^{n}$, then (1.2) is obviously valid for all $u \in W_{p, l o c}^{1}\left(\mathbb{R}^{n}\right)$.

Key words and phrases. Nonlinear elliptic operators, Unbounded domains.

The research was supported by RFBR, grant 11-01-12018-ofi-m-2011. 
For every solution of (1.1), (1.2) we put

$$
M(r ; u)=\underset{S_{r} \cap \Omega}{\operatorname{ess} \sup } u, \quad r>r_{0},
$$

where the restriction of $u$ to $S_{r} \cap \Omega$ is understood in the sense of the trace and the ess sup in the right-hand side is with respect to $(n-1)$-dimensional Lebesgue measure on $S_{r}$.

The research presented to your attention deals with a priori estimates and blowup conditions for solutions of problem (1.1), (1.2). The questions treated below were investigated mainly for nonlinearities of the Emden-Fowler type $g(t)=t^{\lambda}[2$, 6. 7, 9, 10]. Partial cases of inequality (1.1) were also studied in [1, 8]. In our paper, we consider the most general case.

\section{Main Results}

Theorem 2.1. Let

$$
\int_{1}^{\infty}\left(g_{\theta}(t) t\right)^{-1 / p} d t<\infty
$$

and

$$
\int_{r_{0}}^{\infty}\left(r f_{\sigma}(r)\right)^{1 /(p-1)} d r=\infty
$$

for some real numbers $\theta>1$ and $\sigma>1$, then any solution of (1.1), (1.2) is trivial, i.e. $u=0$ almost everywhere in $\Omega$.

Remark 2.1. We remind that, by definition, all solutions of (1.1), (1.2) are nonnegative functions since the domain of the function $g$ is the interval $[0, \infty)$.

Theorem 2.2. Let there be real numbers $\theta>1$ and $\sigma>1$ such that (2.2) is valid and, moreover,

$$
\int_{1}^{\infty}\left(g_{\theta}(t) t\right)^{-1 / p} d t=\infty
$$

Then any nontrivial solution of (1.1), (1.2) satisfies the estimate

$$
\int_{1}^{M(r ; u)}\left(g_{\theta}(t) t\right)^{-1 / p} d t \geq C\left(\int_{r_{0}}^{r}\left(\xi f_{\sigma}(\xi)\right)^{1 /(p-1)} d \xi\right)^{(p-1) / p}
$$

for all sufficiently large $r$, where the constant $C>0$ depends only on $n, p, \theta, \sigma$, $C_{1}$, and $C_{2}$.

Theorem 2.3. In the hypotheses of Theorem 2.2, let the condition

$$
\int_{r_{0}}^{\infty} \min \left\{\left(\xi f_{\sigma}(\xi)\right)^{1 /(p-1)}, q_{\sigma}^{1 / p}(\xi)\right\} d \xi=\infty
$$

be valid instead of (2.2). Then any nontrivial solution of (1.1), (1.2) satisfies the estimate

$$
\begin{gathered}
\int_{1}^{M(r ; u)}\left(g_{\theta}(t) t\right)^{-1 / p} d t+\int_{1}^{M(r ; u)} g_{\theta}^{-1 /(p-1)}(t) d t \\
\geq C \int_{r_{0}}^{r} \min \left\{\left(\xi f_{\sigma}(\xi)\right)^{1 /(p-1)}, q_{\sigma}^{1 / p}(\xi)\right\} d \xi
\end{gathered}
$$

for all sufficiently large $r$, where the constant $C>0$ depends only on $n, p, \theta, \sigma$, $C_{1}$, and $C_{2}$. 
Theorem 2.4. Let there be real numbers $\theta>1$ and $\sigma>1$ such that (2.1) is valid and, moreover,

$$
\int_{r_{0}}^{\infty}\left(r f_{\sigma}(r)\right)^{1 /(p-1)} d r<\infty
$$

Then any solution of (1.1), (1.2) satisfies the estimate

$$
\int_{M(r ; u)}^{\infty}\left(g_{\theta}(t) t\right)^{-1 / p} d t \geq C\left(\int_{r}^{\infty}\left(\xi f_{\sigma}(\xi)\right)^{1 /(p-1)} d \xi\right)^{(p-1) / p}
$$

for all sufficiently large $r$, where the constant $C>0$ depends only on $n, p, \theta, \sigma$, $C_{1}$, and $C_{2}$.

The proof of Theorems 2.1 2.4 is given in Section 3. Now, we consider the case that

$$
|b(x)| \leq \alpha|x|^{k}
$$

with some constants $\alpha$ and $k$ for almost all $x \in \Omega$.

Corollary 2.1. In formula (2.6), let $k \leq-1$. If there are real numbers $\theta>1$ and $\sigma>1$ such that (2.1) is valid and

$$
\int_{r_{0}}^{\infty}\left(r q_{\sigma}(r)\right)^{1 /(p-1)} d r=\infty
$$

then any solution of (1.1), (1.2) is trivial.

Corollary 2.2. In formula (2.6), let $k \leq-1$. Also suppose that conditions (2.3) and (2.7) are valid for some real numbers $\theta>1$ and $\sigma>1$. Then any nontrivial solution of (1.1), (1.2) satisfies the estimate

$$
\int_{1}^{M(r ; u)}\left(g_{\theta}(t) t\right)^{-1 / p} d t \geq C\left(\int_{r_{0}}^{r}\left(\xi q_{\sigma}(\xi)\right)^{1 /(p-1)} d \xi\right)^{(p-1) / p}
$$

for all sufficiently large $r$, where the constant $C>0$ depends only on $n, p, \theta, \sigma$, $C_{1}, C_{2}$, and $\alpha$.

Corollary 2.3. In the hypotheses of Corollary 2.2, let the condition

$$
\int_{r_{0}}^{\infty} \min \left\{\left(\xi q_{\sigma}(\xi)\right)^{1 /(p-1)}, q_{\sigma}^{1 / p}(\xi)\right\} d \xi=\infty
$$

be valid instead of (2.7). Then any nontrivial solution of (1.1), (1.2) satisfies the estimate

$$
\begin{gathered}
\int_{1}^{M(r ; u)}\left(g_{\theta}(t) t\right)^{-1 / p} d t+\int_{1}^{M(r ; u)} g_{\theta}^{-1 /(p-1)}(t) d t \\
\quad \geq C \int_{r_{0}}^{r} \min \left\{\left(\xi q_{\sigma}(\xi)\right)^{1 /(p-1)}, q_{\sigma}^{1 / p}(\xi)\right\} d \xi
\end{gathered}
$$

for all sufficiently large $r$, where the constant $C>0$ depends only on $n, p, \theta, \sigma$, $C_{1}, C_{2}$, and $\alpha$.

Corollary 2.4. In formula (2.6), let $k \leq-1$. If there are real numbers $\theta>1$ and $\sigma>1$ such that (2.1) is valid and

$$
\int_{r_{0}}^{\infty}\left(r q_{\sigma}(r)\right)^{1 /(p-1)} d r<\infty
$$


then any solution of (1.1), (1.2) satisfies the estimate

$$
\int_{M(r ; u)}^{\infty}\left(g_{\theta}(t) t\right)^{-1 / p} d t \geq C\left(\int_{r}^{\infty}\left(\xi q_{\sigma}(\xi)\right)^{1 /(p-1)} d \xi\right)^{(p-1) / p}
$$

for all sufficiently large $r$, where the constant $C>0$ depends only on $n, p, \theta, \sigma$, $C_{1}, C_{2}$, and $\alpha$.

Proof of Corollaries 2.1 2.4 follows immediately from Teorems 2.1 2.4, Really, if $k \leq-1$ in (2.6), then $f_{\sigma}(r) \geq \gamma q_{\sigma}(r)$ for all $r>r_{0}$ and $\sigma>1$, where the constant $\gamma>0$ depends only on $\alpha, k$, and $\sigma$.

In the case of $b=0$, the above statements imply results of paper [3]. Examples given in this paper demonstrate us the precision of Corollaries 2.1 2.4.

Corollary 2.5. In formula (2.6), let $k>-1$. If there are real numbers $\theta>1$ and $\sigma>1$ such that (2.1) is valid and

$$
\int_{r_{0}}^{\infty}\left(r^{-k} q_{\sigma}(r)\right)^{1 /(p-1)} d r=\infty
$$

then any solution of (1.1), (1.2) is trivial.

Proof. The condition $k>-1$ implies the inequality $f_{\sigma}(r) \geq \gamma r^{-k-1} q_{\sigma}(r)$ for all sufficiently large $r$, where the constant $\gamma>0$ depends only on $\alpha, k$, and $\sigma$. Thus, to complete the proof, it remains to use Theorem 2.1 .

Example 2.1. Consider the inequality

$$
\operatorname{div}\left(|D u|^{p-2} D u\right)+b(x)|D u|^{p-1} \geq q(x) u^{\lambda} \quad \text { in } \mathbb{R}^{n},
$$

where $b \in L_{\infty, l o c}\left(\mathbb{R}^{n}\right)$ satisfies relation (2.6) with $k>-1$ and $q \in L_{\infty, l o c}\left(\mathbb{R}^{n}\right)$ is a non-negative function such that

$$
q(x) \sim|x|^{l} \quad \text { as } x \rightarrow \infty
$$

i.e. there exist constants $\alpha_{1}>0$ and $\alpha_{2}>0$ such that

$$
\alpha_{1}|x|^{l} \leq q(x) \leq \alpha_{2}|x|^{l}
$$

for almost all $x$ in a neighborhood of infinity.

By Corollary 2.5, in the case of

$$
\lambda>p-1 \quad \text { and } l \geq k-p+1,
$$

any non-negative solution of (2.9) is trivial.

At the same time, if

$$
\lambda>p-1 \quad \text { and } \quad l<k-p+1
$$

then

$$
u(x)=\left(\max \left\{|x|, r_{0}\right\}\right)^{(k-p+1-l) /(\lambda-p+1)}
$$

is a positive solution of (2.9) for enough large $r_{0}$ with a non-negative function $q \in$ $L_{\infty, l o c}\left(\mathbb{R}^{n}\right)$ satisfying condition (2.10) and a non-negative function $b \in L_{\infty, l o c}\left(\mathbb{R}^{n}\right)$ such that

$$
b(x) \sim|x|^{k} \quad \text { as } x \rightarrow \infty .
$$

Using somewhat more complex reasoning, we can also show that (2.9) has a positive solution for all non-negative functions $b \in L_{\infty, l o c}\left(\mathbb{R}^{n}\right)$ and $q \in L_{\infty, l o c}\left(\mathbb{R}^{n}\right)$ if the first inequality in formula (2.11) does not hold.

Thus, both inequalities in (2.11) are exact. 
Example 2.2. In (2.9), let the non-negative function $q \in L_{\infty, l o c}\left(\mathbb{R}^{n}\right)$ satisfy the relation

$$
q(x) \sim|x|^{k-p+1} \log ^{\mu}|x| \quad \text { as } x \rightarrow \infty .
$$

As in Example 2.1, we assume that (2.6) is fulfilled with $k>-1$.

According to Corollary 2.5, if

$$
\lambda>p-1 \text { and } \mu \geq 1-p,
$$

then any non-negative solution of (2.9) is trivial.

As noted above, the first inequality in formula (2.14) is exact. Let us show that the second one is exact too. In fact, if

$$
\lambda>p-1 \text { and } \mu<1-p,
$$

then

$$
u(x)=\left(\log \max \left\{|x|, r_{0}\right\}\right)^{(1-p-\mu) /(\lambda-p+1)}
$$

is a positive solution of (2.9) for enough large $r_{0}>0$ with some non-negative functions $b \in L_{\infty, l o c}\left(\mathbb{R}^{n}\right)$ and $q \in L_{\infty, l o c}\left(\mathbb{R}^{n}\right)$ satisfying conditions (2.12) and (2.13), respectively.

Example 2.3. Consider the inequality

$$
\operatorname{div}\left(|D u|^{p-2} D u\right)+b(x)|D u|^{p-1} \geq q(x) u^{p-1} \log ^{\lambda}(1+u) \quad \text { in } \mathbb{R}^{n},
$$

where $b \in L_{\infty, l o c}\left(\mathbb{R}^{n}\right)$ satisfies relation (2.6) with $k>-1$ and $q \in L_{\infty, l o c}\left(\mathbb{R}^{n}\right)$ is a non-negative function such that (2.10) holds.

By Corollary 2.5, the conditions

$$
\lambda>p \quad \text { and } l \geq k-p+1,
$$

imply that any non-negative solution of (2.15) is trivial.

On the other hand, if

$$
\lambda>p \quad \text { and } \quad l<k-p+1
$$

then

$$
u(x)=e^{\left(\max \left\{|x|, r_{0}\right\}\right)^{(k-p+1-l) /(\lambda-p+1)}}
$$

is a positive solution of (2.15) for enough large $r_{0}$ with some non-negative functions $q \in L_{\infty, l o c}\left(\mathbb{R}^{n}\right)$ and $b \in L_{\infty, l o c}\left(\mathbb{R}^{n}\right)$ satisfying relations (2.10) and (2.12), respectively. Therefore, the second inequality in formula (2.16) is exact.

The first inequality in (2.16) is also exact. Namely, in the case of $\lambda \leq p$, it can be shown that (2.15) has a positive solution for all non-negative functions $b \in L_{\infty, l o c}\left(\mathbb{R}^{n}\right)$ and $q \in L_{\infty, l o c}\left(\mathbb{R}^{n}\right)$.

Corollary 2.6. Let (2.6) be valid, where $k>-1$. Also suppose that conditions (2.3) and (2.8) hold for some real numbers $\theta>1$ and $\sigma>1$. Then any nontrivial solution of (1.1), (1.2) satisfies the estimate

$$
\int_{1}^{M(r ; u)}\left(g_{\theta}(t) t\right)^{-1 / p} d t \geq C\left(\int_{r_{0}}^{r}\left(\xi^{-k} q_{\sigma}(\xi)\right)^{1 /(p-1)} d \xi\right)^{(p-1) / p}
$$

for all sufficiently large $r$, where the constant $C>0$ depends only on $n, p, \theta, \sigma$, $C_{1}, C_{2}, k$, and $\alpha$.

Proof. We repeat the arguments given in the proof of Corollary 2.5 with Theorem 2.1 replaced by Theorem 2.2 . 
Example 2.4. Consider inequality (2.9), where $\lambda<p-1, b \in L_{\infty, l o c}\left(\mathbb{R}^{n}\right)$, and $q \in L_{\infty, l o c}\left(\mathbb{R}^{n}\right)$ is a non-negative function. We shall assume that condition (2.6) is fulfilled for some $k>-1$.

Let (2.10) hold, where $l>k-p+1$, then in accordance with Corollary 2.6] any nontrivial solution $u \geq 0$ of (2.9) satisfies the estimate

$$
M(r ; u) \geq C r^{(l-k+p-1) /(p-1-\lambda)}
$$

for all enough large $r$, where the constant $C>0$ does not depend of $r$ and $u$.

If we replace (2.10) by (2.13), where $\mu>1-p$, then (2.17) should be replaced by

$$
M(r ; u) \geq C \log (\mu+p-1) /(p-1-\lambda) r
$$

for all enough large $r$, where the constant $C>0$ does not depend of $r$ and $u$.

Example 2.5. Assume that $u \geq 0$ is a nontrivial solution of the inequality

$$
\operatorname{div}\left(|D u|^{p-2} D u\right)+b(x)|D u|^{p-1} \geq q(x) u^{\lambda} \log ^{s}(1+u) \quad \text { in } \mathbb{R}^{n},
$$

where $\lambda<p-1, s \in \mathbb{R}, b \in L_{\infty, l o c}\left(\mathbb{R}^{n}\right)$ and, moreover, condition (2.6) is valid for some $k>-1$. Also let $q \in L_{\infty, l o c}\left(\mathbb{R}^{n}\right)$ be a non-negative function such that (2.10) holds with $l>k-p+1$. Then, according to Corollary 2.6, we have

$$
M(r ; u) \geq C r^{(l-k+p-1) /(p-1-\lambda)} \log ^{s /(p-1-\lambda)} r
$$

for all enough large $r$, where the constant $C>0$ does not depend of $r$ and $u$.

Corollary 2.7. In the hypotheses of Corollary 2.6, let the condition

$$
\int_{r_{0}}^{\infty} \min \left\{\left(\xi^{-k} q_{\sigma}(\xi)\right)^{1 /(p-1)}, q_{\sigma}^{1 / p}(\xi)\right\} d \xi=\infty
$$

be valid instead of (2.8). Then any nontrivial solution of (1.1), (1.2) satisfies the estimate

$$
\begin{gathered}
\int_{1}^{M(r ; u)}\left(g_{\theta}(t) t\right)^{-1 / p} d t+\int_{1}^{M(r ; u)} g_{\theta}^{-1 /(p-1)}(t) d t \\
\geq C \int_{r_{0}}^{r} \min \left\{\left(\xi^{-k} q_{\sigma}(\xi)\right)^{1 /(p-1)}, q_{\sigma}^{1 / p}(\xi)\right\} d \xi
\end{gathered}
$$

for all sufficiently large $r$, where the constant $C>0$ depends only on $n, p, \theta, \sigma$, $C_{1}, C_{2}, k$, and $\alpha$.

Proof. We repeat the arguments given in the proof of Corollary 2.5 with Theorem 2.1 replaced by Theorem 2.3 .

Example 2.6. Consider inequality (2.9) with the critical exponent $\lambda=p-1$. As in Example 2.5, we assume that $b \in L_{\infty, l o c}\left(\mathbb{R}^{n}\right), q \in L_{\infty, l o c}\left(\mathbb{R}^{n}\right)$ is a non-negative function and, moreover, conditions (2.6) and (2.10) are valid, where $k>-1$ and $l>k-p+1$, respectively.

Let $u \geq 0$ be a nontrial solution of (2.9). In the case of $l \leq p k$, applying Corollary 2.7, we have

$$
M(r ; u) \geq e^{C r^{(l-k+p-1) /(p-1)}}
$$

for all enough large $r$, where the constant $C>0$ does not depend of $r$ and $u$. In turn, if $l>p k$, then (2.18) should be replaced by

$$
M(r ; u) \geq e^{C r^{(l+p) / p}}
$$

for all enough large $r$, where the constant $C>0$ does not depend of $r$ and $u$. 
Corollary 2.8. Let (2.6) be valid, where $k>-1$. If there are real numbers $\theta>1$ and $\sigma>1$ such that (2.1) holds and

$$
\int_{r_{0}}^{\infty}\left(r^{-k} q_{\sigma}(r)\right)^{1 /(p-1)} d r<\infty
$$

then any solution of (1.1), (1.2) satisfies the estimate

$$
\int_{M(r ; u)}^{\infty}\left(g_{\theta}(t) t\right)^{-1 / p} d t \geq C\left(\int_{r}^{\infty}\left(\xi^{-k} q_{\sigma}(\xi)\right)^{1 /(p-1)} d \xi\right)^{(p-1) / p}
$$

for all sufficiently large $r$, where the constant $C>0$ depends only on $n, p, \theta, \sigma$, $C_{1}, C_{2}, k$, and $\alpha$.

Proof. We repeat the arguments given in the proof of Corollary 2.5 with Theorem 2.1 replaced by Theorem 2.4 .

Example 2.7. Assume that $u \geq 0$ is a nontrivial solution of inequality (2.9), where $\lambda>p-1, b \in L_{\infty, l o c}\left(\mathbb{R}^{n}\right), q \in L_{\infty, l o c}\left(\mathbb{R}^{n}\right)$ is a non-negative function and, moreover, condition (2.6) is fulfilled for some $k>-1$.

If (2.10) holds with $l<k-p+1$, then in accordance with Corollary 2.8 we obtain

$$
M(r ; u) \leq C r^{(l-k+p-1) /(p-1-\lambda)}
$$

for all enough large $r$, where the constant $C>0$ does not depend of $r$ and $u$.

Now, let condition (2.13) be valid instead of (2.10), where $\mu<1-p$. Then Corollary 2.8 allows us to assert that

$$
M(r ; u) \leq C \log ^{(\mu+p-1) /(p-1-\lambda)} r
$$

for all enough large $r$, where the constant $C>0$ does not depend of $r$ and $u$.

One can easily verify that the estimates given in Examples 2.4 2.7 are exact.

It does not matter for us that the right-hand side of (2.6) is a power function. Theorems 2.1 2.4 remain precise for a wide class of functions $b$.

Assume that

$$
|b(x)| \leq \beta|x|^{k} \log ^{m}|x|
$$

for almost all $x \in \Omega$, where $\beta, k$, and $m$ are some constants and, moreover, either $k>-1$ or $k=-1$ and $m>0$.

Corollary 2.9. Let (2.19) hold. If there are real numbers $\theta>1$ and $\sigma>1$ such that condition (2.1) is valid and

$$
\int_{r_{0}}^{\infty}\left(r^{-k} \log ^{-m} r q_{\sigma}(r)\right)^{1 /(p-1)} d r=\infty
$$

then any solution of (1.1), (1.2) is trivial.

Proof. From (2.19), we have $f_{\sigma}(r) \geq \gamma r^{-k-1} \log ^{-m} r q_{\sigma}(r)$ for all sufficiently large $r$, where the constant $\gamma>0$ depends only on $\beta, k, m$, and $\sigma$. The proof is completed by applying of Theorem 2.1 .

Example 2.8. In (2.9), let $b \in L_{\infty, l o c}\left(\mathbb{R}^{n}\right)$ satisfy condition (2.19) and $q \in L_{\infty, l o c}\left(\mathbb{R}^{n}\right)$ be a non-negative function such that

$$
q(x) \sim|x|^{k-p+1} \quad \text { as } x \rightarrow \infty,
$$

i.e., in formula (2.10), we take the critical exponent $l=k-p+1$. 
According to Corollary 2.9, if

$$
\lambda>p-1 \text { and } m \leq p-1
$$

then any non-negative solution of inequality (2.9) is trivial. As mentioned above, the first condition in (2.21) is exact. Now, we show that the second condition is also exact. Really, let

$$
\lambda>p-1 \text { and } m>p-1 .
$$

By direct calculation, one can verify that

$$
u(x)=\left(\log \max \left\{|x|, r_{0}\right\}\right)^{(m-p+1) /(\lambda-p+1)}
$$

is a positive solution of (2.9) for enough large $r_{0}$, where $q \in L_{\infty, l o c}\left(\mathbb{R}^{n}\right)$ is a nonnegative function such that $(2.20)$ holds and $b \in L_{\infty, l o c}\left(\mathbb{R}^{n}\right)$ is a non-negative function satisfying the relation

$$
b(x) \sim|x|^{k} \log ^{m}|x| \quad \text { as } x \rightarrow \infty .
$$

\section{Proof of Theorems 2.12 .4}

We need a well-known result concerning inequalities of the form

$$
\operatorname{div} A(x, D u) \geq F(x, u, D u) \quad \text { in } \Omega_{R_{0}, R_{1}},
$$

where $0<R_{0}<R_{1} \leq \infty$, the function $A: \Omega \times \mathbb{R}^{n} \rightarrow \mathbb{R}^{n}$ is the same as in (1.1) and $F: \Omega_{R_{0}, R_{1}} \times[0, \infty) \times \mathbb{R}^{n} \rightarrow \mathbb{R}$ satisfies the following conditions: there exist a real number $\sigma>1$ and locally bounded measurable functions $F_{0}:\left[R_{0}, R_{1}\right) \times(0, \infty) \rightarrow$ $[0, \infty)$ and $F_{1}:\left[R_{0}, R_{1}\right) \rightarrow[0, \infty)$ such that

$$
\begin{gathered}
F_{0}(r, t-0)=F_{0}(r, t) \quad \text { for all } R_{0}<r<R_{1}, t>0, \\
F_{0}\left(r, t_{1}\right) \geq F_{0}\left(r, t_{2}\right) \quad \text { for all } R_{0}<r<R_{1}, t_{1} \geq t_{2}>0
\end{gathered}
$$

and

$$
F(x, t, \zeta) \geq \sup _{r \in(|x| / \sigma, \sigma|x|) \cap\left(R_{0}, R_{1}\right)} F_{0}(r, t)-|\zeta|^{p-1} \inf _{r \in(|x| / \sigma, \sigma|x|) \cap\left(R_{0}, R_{1}\right)} F_{1}(r)
$$

for almost all $x \in \Omega_{R_{0}, R_{1}}$ and for all $t \in(0, \infty)$ and $\zeta \in \mathbb{R}^{n}$.

We say that $u$ is a solution of (3.1) if $u \in W_{p}^{1}\left(\Omega_{R_{0}, r}\right) \cap L_{\infty}\left(\Omega_{R_{0}, r}\right), A(x, D u) \in$ $L_{p /(p-1)}\left(\Omega_{R_{0}, r}\right)$, and $F(x, u, D u) \in L_{p /(p-1)}\left(\Omega_{R_{0}, r}\right)$ for any real number $r \in\left(R_{0}, R_{1}\right)$ and, moreover,

$$
-\int_{\Omega_{R_{0}, R_{1}}} A(x, D u) D \varphi d x \geq \int_{\Omega_{R_{0}, R_{1}}} F(x, u, D u) \varphi d x
$$

for any non-negative function $\varphi \in C_{0}^{\infty}\left(\Omega_{R_{0}, R_{1}}\right)$.

According to this definition, any solution of (3.1) must be a non-negative function; otherwise the right-hand side of the last inequality is not well-defined.

Let us denote $\Gamma_{R_{0}, R_{1}}=B_{R_{0}, R_{1}} \cap \partial \Omega$, where $B_{R_{0}, R_{1}}=\left\{x \in \mathbb{R}^{n}: R_{0}<|x|<R_{1}\right\}$. The condition

$$
\left.u\right|_{\Gamma_{R_{0}, R_{1}}}=0
$$

means that $\varphi u \in \stackrel{\circ}{W}_{p}^{1}\left(\Omega_{R_{0}, R_{1}}\right)$ for any $\varphi \in C_{0}^{\infty}\left(B_{R_{0}, R_{1}}\right)$.

We shall assume that $S_{r} \cap \Omega \neq \emptyset$ for all $r \in\left(R_{0}, R_{1}\right)$. 
Lemma 3.1. Suppose that $0<\beta<1$ and $R_{0}<\rho_{0}<\rho_{1}<R_{1}$ are some real numbers. Also let $u$ be a solution of (3.1), (3.3) such that $M(\cdot ; u)$ is a non-decreasing function on $\left(R_{0}, R_{1}\right)$ satisfying the relation $M\left(R_{0}+0 ; u\right)>0$. If $\beta^{1 / 2} M\left(\rho_{1} ; u\right) \leq M\left(\rho_{0} ; u\right)$ and $\sigma^{2} \rho_{0} \geq \rho_{1}$, then

$$
M\left(\rho_{1} ; u\right)-M\left(\rho_{0} ; u\right) \geq \gamma \min \left\{\left(\rho_{1}-\rho_{0}\right)^{p /(p-1)}, \frac{\rho_{1}-\rho_{0}}{\lambda^{1 /(p-1)}}\right\} F_{0}^{1 /(p-1)}\left(s, \beta M\left(\rho_{1} ; u\right)\right)
$$

for all $s \in\left[\rho_{1} / \sigma, \sigma \rho_{0}\right] \cap\left(R_{0}, R_{1}\right)$, where

$$
\lambda=\inf _{\left[\rho_{1} / \sigma, \sigma \rho_{0}\right] \cap\left(R_{0}, R_{1}\right)} F_{1}
$$

and the constant $\gamma>0$ depends only on $n, p, C_{1}, C_{2}$, and $\beta$.

The proof is given in paper [4, Lemma 3.1].

Remark 3.1. If $u$ is a solution of (1.1), (1.2), then

$$
M(r ; u)=\underset{B_{r} \cap \Omega}{\operatorname{ess} \sup } u, \quad r \in\left(R_{0}, R_{1}\right),
$$

in accordance with the maximum principle. Hence, $M(\cdot ; u)$ is a non-decreasing function on $\left(R_{0}, R_{1}\right)$. In addition, we have $M(r ; u)=M(r-0 ; u)$ for all $r \in$ $\left(R_{0}, R_{1}\right)$.

Lemma 3.2. Let $0<R_{0}<R_{1}<\infty$ and, moreover, $u$ be a solution of (1.1), (1.2) such that $M\left(R_{0}+0 ; u\right)>0$ and $\beta^{1 / 2} M\left(R_{1}-0 ; u\right) \leq M\left(R_{0}+0 ; u\right)$ for some real number $0<\beta<1$. Then

$$
M\left(R_{1}-0 ; u\right)-M\left(R_{0}+0 ; u\right) \geq \gamma \min \left\{\left(R_{1}-R_{0}\right)^{p /(p-1)}, \frac{R_{1}-R_{0}}{\mu^{1 /(p-1)}}\right\} Q G,
$$

where $\gamma>0$ is the constant of Lemma 3.1,

$$
\begin{gathered}
Q=\operatorname{essinf}_{\Omega_{R_{0}, R_{1}}} q^{1 /(p-1)}, \\
G=\min _{\left[\beta^{2} M\left(R_{1}-0 ; u\right), M\left(R_{1}-0 ; u\right)\right]} g^{1 /(p-1)},
\end{gathered}
$$

and

$$
\mu=\underset{\Omega_{R_{0}, R_{1}}}{\operatorname{ess} \sup }|b|
$$

Proof. As mentioned in Remark 3.1, $M(\cdot ; u)$ is a non-decreasing function on the interval $\left(R_{0}, R_{1}\right)$. Also it can be seen that

$$
M\left(R_{1}-0 ; u\right)=\operatorname{ess~sup}_{B_{R_{1}} \cap \Omega} u .
$$

In particular, $u(x) \leq M\left(R_{1}-0 ; u\right)$ for almost all $x \in B_{R_{1}} \cap \Omega$.

Let us denote $I=\left(\beta^{2} M\left(R_{1}-0 ; u\right), \infty\right)$. The function $u$ is a solution of (3.1), (3.3), where

$$
F(x, t, \zeta)=\chi_{I}(t) Q^{p-1} G^{p-1}-\mu|\zeta|^{p-1} .
$$

Here, $\chi_{I}$ is the characteristic function of the set $I$, i.e.

$$
\chi_{I}(t)= \begin{cases}1, & t \in I \\ 0, & t \notin I\end{cases}
$$


Putting further $F_{0}(r, t)=\chi_{I}(t) Q^{p-1} G^{p-1}$ and $F_{1}(r)=\mu$, one can verify that (3.2) is fulfilled for any $\sigma>1$. Thus, applying Lemma 3.1, we obtain

$$
M\left(\rho_{1} ; u\right)-M\left(\rho_{0} ; u\right) \geq \gamma \min \left\{\left(\rho_{1}-\rho_{0}\right)^{p /(p-1)}, \frac{\rho_{1}-\rho_{0}}{\mu^{1 /(p-1)}}\right\} Q G
$$

for all $R_{0}<\rho_{0}<\rho_{1}<R_{1}$, whence (3.4) follows at once.

The proof is completed.

Lemma 3.3. Let $u$ be a solution of (1.1), (1.2). If $r_{0} \leq r_{1}<r_{2}, \eta>1$, and $\tau>1$ are real numbers such that $\eta M\left(r_{1}+0 ; u\right) \leq M\left(r_{2} ; u\right), r_{2} \leq \tau r_{1}$, and $M\left(r_{1}+0 ; u\right)>$ 0 , then at least one of the following two inequalities is valid:

$$
\begin{gathered}
\int_{M\left(r_{1}+0 ; u\right)}^{M\left(r_{2} ; u\right)}\left(g_{\eta}(t) t\right)^{-1 / p} d t \geq C \int_{r_{1}}^{r_{2}} q_{\tau}^{1 / p}(\xi) d \xi, \\
\int_{M\left(r_{1}+0 ; u\right)}^{M\left(r_{2} ; u\right)} g_{\eta}^{-1 /(p-1)}(t) d t \geq C \int_{r_{1}}^{r_{2}}\left(\xi f_{\tau}(\xi)\right)^{1 /(p-1)} d \xi,
\end{gathered}
$$

where the constant $C>0$ depends only on $n, p, \eta, C_{1}$, and $C_{2}$.

Proof. The function $M(\cdot ; u)$ does not decrease on the interval $\left[r_{1}, r_{2}\right]$ and, moreover, $M(r ; u)=M(r-0 ; u)$ for all $r \in\left(r_{1}, r_{2}\right]$. By induction, we construct the finite sequence of real numbers $\rho_{0}<\rho_{1}<\ldots<\rho_{k}$. Let us take $\rho_{0}=r_{2}$. Now, assume that $\rho_{i}$ is already known. We put

$$
\rho_{i+1}=\inf \left\{\xi \in\left(r_{1}, \rho_{i}\right): M(\xi ; u)>\eta^{-1 / 8} M\left(\rho_{i} ; u\right)\right\} .
$$

If $\rho_{i+1}=r_{1}$, then we take $k=i+1$ and stop. It is obvious that this procedure must terminate at a finite step. Since $M(\cdot ; u)$ is a semicontinuous function, one can claim that $\left\{\xi \in\left(r_{1}, \rho_{i}\right): M(\xi ; u)>\eta^{-1 / 8} M\left(\rho_{i} ; u\right)\right\} \neq \emptyset$ for any $i \in\{0, \ldots, k-1\}$. Thus, the right-hand side of (3.7) is well defined. In so doing, we have

$$
\eta^{-1 / 8} M\left(\rho_{i} ; u\right) \leq M\left(\rho_{i+1}+0 ; u\right), \quad i=0, \ldots, k-1
$$

and

$$
M\left(\rho_{i+1} ; u\right) \leq \eta^{-1 / 8} M\left(\rho_{i} ; u\right), \quad i=0, \ldots, k-2 .
$$

For any $i \in\{0, \ldots, k-1\}$, according to Lemma 3.2, at least one of the following two estimates holds:

$$
\begin{gathered}
M\left(\rho_{i} ; u\right)-M\left(\rho_{i+1}+0 ; u\right) \geq \varkappa_{1}\left(\rho_{i}-\rho_{i+1}\right)^{p /(p-1)} Q_{i} G_{i} \\
M\left(\rho_{i} ; u\right)-M\left(\rho_{i+1}+0 ; u\right) \geq \frac{\varkappa_{1}\left(\rho_{i}-\rho_{i+1}\right) Q_{i} G_{i}}{\mu_{i}^{1 /(p-1)}},
\end{gathered}
$$

where

$$
\begin{aligned}
& Q_{i}=\underset{\Omega_{\rho_{i+1}, \rho_{i}}}{\operatorname{essinf}} q^{1 /(p-1)}, \\
& G_{i}=\min _{\left[\eta^{-1 / 2} M\left(\rho_{i} ; u\right), M\left(\rho_{i} ; u\right)\right]} g^{1 /(p-1)}, \\
& \mu_{i}=\underset{\Omega_{\rho_{i+1}, \rho_{i}}}{\operatorname{ess} \sup }|b|,
\end{aligned}
$$

and $\varkappa_{1}>0$ is some constant depending only on $n, p, C_{1}, C_{2}$, and $\eta$.

By $\Xi_{1}$ we denote the set of integers $i \in\{0, \ldots, k-1\}$ for which (3.10) is fulfilled. Also let $\Xi_{2}=\{0, \ldots, k-1\} \backslash \Xi_{1}$. 
At first, we assume that

$$
\sum_{i \in \Xi_{1}}\left(\rho_{i}-\rho_{i+1}\right) \geq \frac{r_{2}-r_{1}}{2}
$$

Formula (3.10) implies the relation

$$
\left(\frac{M\left(\rho_{i} ; u\right)-M\left(\rho_{i+1}+0 ; u\right)}{G_{i}}\right)^{(p-1) / p} \geq \varkappa_{1}^{(p-1) / p}\left(\rho_{i}-\rho_{i+1}\right) Q_{i}^{(p-1) / p},
$$

whence, taking into account (3.8), we obtain

$$
\frac{M\left(\rho_{i} ; u\right)-\eta^{-1 / 8} M\left(\rho_{i} ; u\right)}{G_{i}^{(p-1) / p} M^{1 / p}\left(\rho_{i} ; u\right)} \geq \varkappa_{2}\left(\rho_{i}-\rho_{i+1}\right) Q_{i}^{(p-1) / p}
$$

for any $i \in \Xi_{1}$, where $\varkappa_{2}=\left(1-\eta^{-1 / 8}\right)^{1 / p} \varkappa_{1}^{(p-1) / p}$. Combining this with the inequality

$$
\int_{\eta^{-1 / 8} M\left(\rho_{i} ; u\right)}^{M\left(\rho_{i} ; u\right)}\left(g_{\eta}(t) t\right)^{-1 / p} d t \geq \frac{M\left(\rho_{i} ; u\right)-\eta^{-1 / 8} M\left(\rho_{i} ; u\right)}{G_{i}^{(p-1) / p} M^{1 / p}\left(\rho_{i} ; u\right)},
$$

one can conclude that

$$
\int_{\eta^{-1 / 8} M\left(\rho_{i} ; u\right)}^{M\left(\rho_{i} ; u\right)}\left(g_{\eta}(t) t\right)^{-1 / p} d t \geq \varkappa_{2}\left(\rho_{i}-\rho_{i+1}\right) Q_{i}^{(p-1) / p}
$$

for any $i \in \Xi_{1}$. Summing the last estimate over all $i \in \Xi_{1} \cap\{0, \ldots, k-2\}$, we have

$$
\sum_{i \in \Xi_{1} \cap\{0, \ldots, k-2\}} \int_{\eta^{-1 / 8} M\left(\rho_{i} ; u\right)}^{M\left(\rho_{i} ; u\right)}\left(g_{\eta}(t) t\right)^{-1 / p} d t \geq \varkappa_{2} \operatorname{sss}_{\Omega_{r_{1}, r_{2}}}^{\operatorname{enf}} q^{1 / p} \sum_{i \in \Xi_{1} \cap\{0, \ldots, k-2\}}\left(\rho_{i}-\rho_{i+1}\right) .
$$

By (3.9), this implies that

$$
\int_{M\left(\rho_{k-1} ; u\right)}^{M\left(\rho_{0} ; u\right)}\left(g_{\eta}(t) t\right)^{-1 / p} d t \geq \varkappa_{2} \underset{\Omega_{r_{1}, r_{2}}}{\operatorname{ess} \inf } q^{1 / p} \sum_{i \in \Xi_{1} \cap\{0, \ldots, k-2\}}\left(\rho_{i}-\rho_{i+1}\right) .
$$

If $k-1 \notin \Xi_{1}$, then combining (3.12) and (3.13), we immediately obtain (3.5). Let $k-1 \in \Xi_{1}$. Putting $i=k-1$ in formula (3.10), we obviously have

$$
\left(\frac{M\left(\rho_{k-1} ; u\right)-M\left(\rho_{k}+0 ; u\right)}{G_{k-1}}\right)^{(p-1) / p} \geq \varkappa_{1}^{(p-1) / p}\left(\rho_{k-1}-\rho_{k}\right) Q_{k-1}^{(p-1) / p} .
$$

At the same time, $M\left(\rho_{k-1} ; u\right) \leq \eta^{1 / 8} M\left(\rho_{k}+0 ; u\right)$ according to (3.8); therefore, one can assert that

$$
\frac{\eta^{1 / 8} M\left(\rho_{k}+0 ; u\right)-M\left(\rho_{k}+0 ; u\right)}{G_{k-1}^{(p-1) / p} M^{1 / p}\left(\rho_{k}+0 ; u\right)} \geq \varkappa_{3}\left(\rho_{k-1}-\rho_{k}\right) Q_{k-1}^{(p-1) / p},
$$

where $\varkappa_{3}=\left(\eta^{1 / 8}-1\right)^{1 / p} \varkappa_{1}^{(p-1) / p}$. Since

$$
\int_{M\left(\rho_{k}+0 ; u\right)}^{\eta^{1 / 8} M\left(\rho_{k}+0 ; u\right)}\left(g_{\eta}(t) t\right)^{-1 / p} d t \geq \frac{\eta^{1 / 8} M\left(\rho_{k}+0 ; u\right)-M\left(\rho_{k}+0 ; u\right)}{\eta^{p / 8} G_{k-1}^{(p-1) / p} M^{1 / p}\left(\rho_{k}+0 ; u\right)},
$$

formula (3.14) implies the estimate

$$
\int_{M\left(\rho_{k}+0 ; u\right)}^{\eta^{1 / 8} M\left(\rho_{k}+0 ; u\right)}\left(g_{\eta}(t) t\right)^{-1 / p} d t \geq \varkappa_{4}\left(\rho_{k-1}-\rho_{k}\right) Q_{k-1}^{(p-1) / p}
$$


where $\varkappa_{4}=\eta^{-p / 8} \varkappa_{3}$. Summing this with (3.13), we obtain

$$
\int_{M\left(r_{1}+0 ; u\right)}^{M\left(r_{2} ; u\right)}\left(g_{\eta}(t) t\right)^{-1 / p} d t \geq \varkappa_{5} \underset{\Omega_{r_{1}, r_{2}}}{\operatorname{essinf}} q^{1 / p} \sum_{i \in \Xi_{1}}\left(\rho_{i}-\rho_{i+1}\right),
$$

where $\varkappa_{5}=\min \left\{\varkappa_{2}, \varkappa_{4}\right\} / 2$. Finally, combining the last inequality and (3.12), we again derive (3.5).

Now, assume that (3.12) is not valid. Thus, we have

$$
\sum_{i \in \Xi_{2}}\left(\rho_{i}-\rho_{i+1}\right) \geq \frac{r_{2}-r_{1}}{2}
$$

Let $i \in \Xi_{2}$. From (3.11), it follows that

$$
\frac{M\left(\rho_{i} ; u\right)-M\left(\rho_{i+1}+0 ; u\right)}{G_{i}} \geq \frac{\varkappa_{1}\left(\rho_{i}-\rho_{i+1}\right) Q_{i}}{\mu_{i}^{1 /(p-1)}},
$$

whence, taking into account the relation

$$
\int_{M\left(\rho_{i+1}+0 ; u\right)}^{M\left(\rho_{i} ; u\right)} g_{\eta}^{-1 /(p-1)}(t) d t \geq \frac{M\left(\rho_{i} ; u\right)-M\left(\rho_{i+1}+0 ; u\right)}{G_{i}},
$$

we obtain

$$
\int_{M\left(\rho_{i+1}+0 ; u\right)}^{M\left(\rho_{i} ; u\right)} g_{\eta}^{-1 /(p-1)}(t) d t \geq \frac{\varkappa_{1}\left(\rho_{i}-\rho_{i+1}\right) Q_{i}}{\mu_{i}^{1 /(p-1)}} .
$$

Summing (3.17) over all $i \in \Xi_{2}$, one can conclude that

$$
\int_{M\left(r_{1}+0 ; u\right)}^{M\left(r_{2} ; u\right)} g_{\eta}^{-1 /(p-1)}(t) d t \geq \frac{\varkappa_{1} \underset{\Omega_{r_{1}, r_{2}}}{\operatorname{essinf}} q^{1 /(p-1)}}{\operatorname{ess~sup}_{\Omega_{r_{1}, r_{2}}}|b|^{1 /(p-1)}} \sum_{i \in \Xi_{2}}\left(\rho_{i}-\rho_{i+1}\right) .
$$

By (3.15), this immediately implies (3.6).

The proof is completed.

Lemma 3.4. Let $u$ be a solution of (1.1), (1.2). If $r_{0} \leq r_{1}<r_{2}, \eta>1$, and $\tau>1$ are real numbers such that $M\left(r_{2} ; u\right) \leq \eta M\left(r_{1}+0 ; u\right), \tau^{1 / 2} r_{1} \leq r_{2}$, and $M\left(r_{1}+0 ; u\right)>0$, then estimate (3.6) holds, where the constant $C>0$ depends only on $n, p, \eta, \tau, C_{1}$, and $C_{2}$.

Proof. Consider the maximal integer $k$ satisfying the condition $\tau^{k / 2} r_{1} \leq r_{2}$. We put $\rho_{i}=\tau^{-i / 2} r_{2}, i=0, \ldots, k-1$, and $\rho_{k}=r_{1}$. From Lemma 3.2, it follows that at least one of inequalities (3.10), (3.11) is valid for any $i \in\{0, \ldots, k-1\}$. If (3.10) holds for some $i \in\{0, \ldots, k-1\}$, then we have

$$
\frac{M\left(\rho_{i} ; u\right)-M\left(\rho_{i+1}+0 ; u\right)}{G_{i}} \geq \varkappa_{1}\left(\rho_{i}-\rho_{i+1}\right)^{p /(p-1)} Q_{i} .
$$

Since $\rho_{i}-\rho_{i+1} \geq\left(1-\tau^{-1 / 2}\right) \rho_{i}$, this implies the estimate

$$
\frac{M\left(\rho_{i} ; u\right)-M\left(\rho_{i+1}+0 ; u\right)}{G_{i}} \geq \varkappa_{6}\left(\rho_{i}-\rho_{i+1}\right) \rho_{i}^{1 /(p-1)} Q_{i},
$$

where the constant $\varkappa_{6}>0$ depends only on $n, p, \eta, \tau, C_{1}$, and $C_{2}$. Consequently, taking into account (3.16) and the fact that

$$
\int_{\rho_{i+1}}^{\rho_{i}}\left(\xi f_{\tau}(\xi)\right)^{1 /(p-1)} d \xi \leq\left(\rho_{i}-\rho_{i+1}\right) \rho_{i}^{1 /(p-1)} Q_{i}
$$


we obtain

$$
\int_{M\left(\rho_{i+1}+0 ; u\right)}^{M\left(\rho_{i} ; u\right)} g_{\eta}^{-1 /(p-1)}(t) d t \geq \varkappa_{6} \int_{\rho_{i+1}}^{\rho_{i}}\left(\xi f_{\tau}(\xi)\right)^{1 /(p-1)} d \xi .
$$

In turn, if (3.11) is fulfilled for some $i \in\{0, \ldots, k-1\}$, then

$$
\frac{M\left(\rho_{i} ; u\right)-M\left(\rho_{i+1}+0 ; u\right)}{G_{i}} \geq \frac{\varkappa_{1}\left(\rho_{i}-\rho_{i+1}\right) Q_{i}}{\mu_{i}^{1 /(p-1)}},
$$

whence in accodance with (3.16) and the evident inequality

$$
\int_{\rho_{i+1}}^{\rho_{i}}\left(\xi f_{\tau}(\xi)\right)^{1 /(p-1)} d \xi \leq \frac{\left(\rho_{i}-\rho_{i+1}\right) Q_{i}}{\mu_{i}^{1 /(p-1)}}
$$

formula (3.18) follows again.

To complete the proof, it remains to sum (3.18) over all $i \in\{0, \ldots, k-1\}$.

Lemma 3.5. Let $u$ be a solution of (1.1), (1.2). If $r_{0} \leq r_{1}<r_{2}, \theta>1$, and $\sigma>1$ are real numbers such that $\theta M\left(r_{1}+0 ; u\right) \leq M\left(r_{2} ; u\right), r_{2} \geq \sigma r_{1}$, and $M\left(r_{1}+0 ; u\right)>$ 0 , then

$$
\int_{M\left(r_{1}+0 ; u\right)}^{M\left(r_{2} ; u\right)}\left(g_{\theta}(t) t\right)^{-1 / p} d t \geq C\left(\int_{r_{1}}^{r_{2}}\left(\xi f_{\sigma}(\xi)\right)^{1 /(p-1)} d \xi\right)^{(p-1) / p}
$$

where the constant $C>0$ depends only on $n, p, \theta, \sigma, C_{1}$, and $C_{2}$.

Proof. We denote $\tau=\sigma^{1 / 2}$ and $\eta=\theta^{1 / 2}$. Take the maximal integer $k$ satisfying the condition $\tau^{k / 2} r_{1} \leq r_{2}$. As in the proof of Lemma 3.4, we put $\rho_{i}=\tau^{-i / 2} r_{2}$, $i=0, \ldots, k-1$, and $\rho_{k}=r_{1}$.

According to Lemmas 3.3 and 3.4, for any $i \in\{0, \ldots, k-1\}$ at least one of the following two inequalities holds:

$$
\begin{gathered}
\int_{M\left(\rho_{i+1}+0 ; u\right)}^{M\left(\rho_{i} ; u\right)}\left(g_{\eta}(t) t\right)^{-1 / p} d t \geq \varkappa_{7} \int_{\rho_{i+1}}^{\rho_{i}} q_{\tau}^{1 / p}(\xi) d \xi, \\
\int_{M\left(\rho_{i+1}+0 ; u\right)}^{M\left(\rho_{i} ; u\right)} g_{\eta}^{-1 /(p-1)}(t) d t \geq \varkappa_{7} \int_{\rho_{i+1}}^{\rho_{i}}\left(\xi f_{\tau}(\xi)\right)^{1 /(p-1)} d \xi,
\end{gathered}
$$

where the constant $\varkappa_{7}>0$ depends only on $n, p, \eta, \tau, C_{1}$, and $C_{2}$.

By $\Xi_{1}$ we mean the set of integers $i \in\{0, \ldots, k-1\}$ for which (3.20) is fulfilled. Also put $\Xi_{2}=\{0, \ldots, k-1\} \backslash \Xi_{1}$.

At first, let

$$
\sum_{i \in \Xi_{1}} \int_{\rho_{i+1}}^{\rho_{i}}\left(\xi f_{\sigma}(\xi)\right)^{1 /(p-1)} d \xi \geq \frac{1}{2} \int_{r_{1}}^{r_{2}}\left(\xi f_{\sigma}(\xi)\right)^{1 /(p-1)} d \xi
$$

Summing (3.20) over all $i \in \Xi_{1}$, we have

$$
\int_{M\left(r_{1}+0 ; u\right)}^{M\left(r_{2} ; u\right)}\left(g_{\eta}(t) t\right)^{-1 / p} d t \geq \varkappa_{7} \sum_{i \in \Xi_{1}} \int_{\rho_{i+1}}^{\rho_{i}} q_{\tau}^{1 / p}(\xi) d \xi
$$

For any $i \in\{0, \ldots, k-1\}$ there exists $\xi_{i} \in\left(\rho_{i+1}, \rho_{i}\right)$ such that

$$
\int_{\rho_{i+1}}^{\rho_{i}} q_{\tau}^{1 / p}(\xi) d \xi \geq\left(\rho_{i}-\rho_{i+1}\right) q_{\tau}^{1 / p}\left(\xi_{i}\right)
$$


Thus,

$$
\begin{aligned}
\left(\sum_{i \in \Xi_{1}} \int_{\rho_{i+1}}^{\rho_{i}} q_{\tau}^{1 / p}(\xi) d \xi\right)^{p /(p-1)} & \geq \sum_{i \in \Xi_{1}}\left(\int_{\rho_{i+1}}^{\rho_{i}} q_{\tau}^{1 / p}(\xi) d \xi\right)^{p /(p-1)} \\
& \geq \sum_{i \in \Xi_{1}}\left(\rho_{i}-\rho_{i+1}\right)^{p /(p-1)} q_{\tau}^{1 /(p-1)}\left(\xi_{i}\right)
\end{aligned}
$$

whence, taking into account the fact that

$$
\begin{aligned}
\left(\rho_{i}-\rho_{i+1}\right)^{p /(p-1)} q_{\tau}^{1 /(p-1)}\left(\xi_{i}\right) & \geq\left(1-\tau^{-1 / 2}\right)^{1 /(p-1)}\left(\rho_{i}-\rho_{i+1}\right)\left(\rho_{i} q_{\tau}\left(\xi_{i}\right)\right)^{1 /(p-1)} \\
& \geq\left(1-\tau^{-1 / 2}\right)^{1 /(p-1)} \int_{\rho_{i+1}}^{\rho_{i}}\left(\xi f_{\sigma}(\xi)\right)^{1 /(p-1)} d \xi
\end{aligned}
$$

for any $i \in \Xi_{1}$, we obtain

$$
\left(\sum_{i \in \Xi_{1}} \int_{\rho_{i+1}}^{\rho_{i}} q_{\tau}^{1 / p}(\xi) d \xi\right)^{p /(p-1)} \geq\left(1-\tau^{-1 / 2}\right)^{1 /(p-1)} \sum_{i \in \Xi_{1}} \int_{\rho_{i+1}}^{\rho_{i}}\left(\xi f_{\sigma}(\xi)\right)^{1 /(p-1)} d \xi
$$

The last estimate, (3.22), and (3.23) immediately imply (3.19).

Now, assume that (3.22) is not valid. In this case, we obviously have

$$
\sum_{i \in \Xi_{2}} \int_{\rho_{i+1}}^{\rho_{i}}\left(\xi f_{\sigma}(\xi)\right)^{1 /(p-1)} d \xi \geq \frac{1}{2} \int_{r_{1}}^{r_{2}}\left(\xi f_{\sigma}(\xi)\right)^{1 /(p-1)} d \xi
$$

For every $i \in \Xi_{2}$ inequality (3.21) holds. Consequently,

$$
\int_{M\left(r_{1}+0 ; u\right)}^{M\left(r_{2} ; u\right)} g_{\eta}^{-1 /(p-1)}(t) d t \geq \varkappa_{7} \sum_{i \in \Xi_{2}} \int_{\rho_{i+1}}^{\rho_{i}}\left(\xi f_{\tau}(\xi)\right)^{1 /(p-1)} d \xi
$$

Let us show that

$$
\left(\int_{M\left(r_{1}+0 ; u\right)}^{M\left(r_{2} ; u\right)}\left(g_{\theta}(t) t\right)^{-1 / p} d t\right)^{p /(p-1)} \geq \varkappa_{8} \int_{M\left(r_{1}+0 ; u\right)}^{M\left(r_{2} ; u\right)} g_{\eta}^{-1 /(p-1)}(t) d t
$$

where the constant $\varkappa_{8}>0$ depends only on $p$ and $\theta$.

Really, take the maximal integer $l$ satisfying the condition $\eta^{l / 2} M\left(r_{1}+0 ; u\right) \leq$ $M\left(r_{2} ; u\right)$. We put $m_{i}=\eta^{-i / 2} M\left(r_{2} ; u\right), i=0, \ldots, l-1$, and $m_{l}=M\left(r_{1}+0 ; u\right)$.

For any $i \in\{0, \ldots, l-1\}$ there exists $t_{i} \in\left(m_{i+1}, m_{i}\right)$ such that

$$
\int_{m_{i+1}}^{m_{i}}\left(g_{\theta}(t) t\right)^{-1 / p} d t \geq\left(m_{i}-m_{i+1}\right)\left(g_{\theta}\left(t_{i}\right) t_{i}\right)^{-1 / p}
$$

Hence, we have

$$
\begin{aligned}
\left(\int_{M\left(r_{1}+0 ; u\right)}^{M\left(r_{2} ; u\right)}\left(g_{\theta}(t) t\right)^{-1 / p} d t\right)^{p /(p-1)} & =\left(\sum_{i=0}^{l-1} \int_{m_{i+1}}^{m_{i}}\left(g_{\theta}(t) t\right)^{-1 / p} d t\right)^{p /(p-1)} \\
& \geq \sum_{i=0}^{l-1}\left(\int_{m_{i+1}}^{m_{i}}\left(g_{\theta}(t) t\right)^{-1 / p} d t\right)^{p /(p-1)} \\
& \geq \sum_{i=0}^{l-1}\left(m_{i}-m_{i+1}\right)^{p /(p-1)}\left(g_{\theta}\left(t_{i}\right)_{i}\right)^{-1 /(p-1)}
\end{aligned}
$$


Since

$$
\begin{aligned}
\left(m_{i}-m_{i+1}\right)^{p /(p-1)}\left(g_{\theta}\left(t_{i}\right) t_{i}\right)^{-1 /(p-1)} & \geq\left(1-\eta^{-1 / 2}\right)^{1 /(p-1)}\left(m_{i}-m_{i+1}\right) g_{\theta}^{-1 /(p-1)}\left(t_{i}\right) \\
& \geq\left(1-\eta^{-1 / 2}\right)^{1 /(p-1)} \int_{m_{i+1}}^{m_{i}} g_{\eta}^{-1 /(p-1)}(t) d t
\end{aligned}
$$

for all $i \in\{0, \ldots, l-1\}$, this implies the estimate

$$
\left(\int_{M\left(r_{1}+0 ; u\right)}^{M\left(r_{2} ; u\right)}\left(g_{\theta}(t) t\right)^{-1 / p} d t\right)^{p /(p-1)} \geq\left(1-\eta^{-1 / 2}\right)^{1 /(p-1)} \sum_{i=0}^{l-1} \int_{m_{i+1}}^{m_{i}} g_{\eta}^{-1 /(p-1)}(t) d t
$$

from which (3.26) follows at once.

To complete the proof, it remains to combine (3.24), (3.25), and (3.26).

Proof of Theorem 2.1. Let $u$ be a nontrivial solution of (1.1), (1.2). There obviously exists a real number $r_{1}>r_{0}$ such that $M\left(r_{1} ; u\right)>0$. By the maximum principle, $M(\cdot ; u)$ is a non-decreasing function on the interval $\left(r_{0}, \infty\right)$; therefore, $M\left(r_{1}+0 ; u\right) \geq M\left(r_{1} ; u\right)>0$.

At first, assume that

$$
\lim _{r \rightarrow \infty} M(r ; u)=M(\infty ; u)<\infty .
$$

Taking the real number $r_{1}$ large enough, we obtain $M\left(r_{1}+0 ; u\right) \leq \theta M(r ; u)$ for all $r>r_{1}$. Thus,

$$
\int_{M\left(r_{1}+0 ; u\right)}^{M(\infty ; u)} g_{\theta}^{-1 /(p-1)}(t) d t \geq C \int_{r_{1}}^{\infty}\left(\xi f_{\sigma}(\xi)\right)^{1 /(p-1)} d \xi
$$

by Lemma 3.4, where the constant $C>0$ depends only on $n, p, \theta, \sigma, C_{1}$, and $C_{2}$. This contradicts condition (2.2).

Now, let

$$
\lim _{r \rightarrow \infty} M(r ; u)=\infty
$$

then Lemma 3.5 allows us to assert that

$$
\int_{M\left(r_{1}+0 ; u\right)}^{\infty}\left(g_{\theta}(t) t\right)^{-1 / p} d t \geq C\left(\int_{r_{1}}^{\infty}\left(\xi f_{\sigma}(\xi)\right)^{1 /(p-1)} d \xi\right)^{(p-1) / p}
$$

with some constant $C>0$ dependind only on $n, p, \theta, \sigma, C_{1}$, and $C_{2}$. In turn, this contradicts conditions (2.1) and (2.2).

The proof is completed.

Proof of Theorem 2.2. Let $u$ be a nontrivial solution of (1.1), (1.2). As in the proof of Theorem 2.1, we take a real number $r_{1}>r_{0}$ such that $M\left(r_{1} ; u\right)>0$. If (3.27) holds, then Lemma 3.4 implies inequality (3.28) which contradicts (2.2). Consequently, one can assume that (3.29) is fulfilled.

According to Lemma 3.5.

$$
\int_{M\left(r_{1}+0 ; u\right)}^{M(r ; u)}\left(g_{\theta}(t) t\right)^{-1 / p} d t \geq C\left(\int_{r_{1}}^{r}\left(\xi f_{\sigma}(\xi)\right)^{1 /(p-1)} d \xi\right)^{(p-1) / p}
$$

for all sufficiently large $r$, where the constant $C>0$ depends only on $n, p, \theta, \sigma$, $C_{1}$, and $C_{2}$. In so doing, from (2.2) and (2.3), it follows that

$$
\int_{r_{1}}^{r}\left(\xi f_{\sigma}(\xi)\right)^{1 /(p-1)} d \xi \geq \frac{1}{2} \int_{r_{0}}^{r}\left(\xi f_{\sigma}(\xi)\right)^{1 /(p-1)} d \xi
$$


and

$$
\int_{1}^{M(r ; u)}\left(g_{\theta}(t) t\right)^{-1 / p} d t \geq \frac{1}{2} \int_{M\left(r_{1}+0 ; u\right)}^{M(r ; u)}\left(g_{\theta}(t) t\right)^{-1 / p} d t
$$

for all sufficiently large $r$.

Thus, combining the last three estimates, we complete the proof.

Proof of Theorem 2.3. Let $u$ be a nontrivial solution of (1.1), (1.2). We take a real number $r_{1}>r_{0}$ such that $M\left(r_{1} ; u\right)>0$. Also assume that $r>r_{1}$ is a real number satisfying the condition $\sigma r_{1}<r$ and, moreover, $k$ is the maximal integer for which $\sigma^{k / 2} r_{1} \leq r$. We put $\rho_{i}=\sigma^{-i / 2} r, i=0, \ldots, k-1$, and $\rho_{k}=r_{1}$.

By Lemmas 3.3 and 3.4, for any $i \in\{0, \ldots, k-1\}$ at least one of the following two inequalities is valid:

$$
\begin{gathered}
\int_{M\left(\rho_{i+1}+0 ; u\right)}^{M\left(\rho_{i} ; u\right)}\left(g_{\theta}(t) t\right)^{-1 / p} d t \geq C \int_{\rho_{i+1}}^{\rho_{i}} q_{\sigma}^{1 / p}(\xi) d \xi \\
\int_{M\left(\rho_{i+1}+0 ; u\right)}^{M\left(\rho_{i} ; u\right)} g_{\theta}^{-1 /(p-1)}(t) d t \geq C \int_{\rho_{i+1}}^{\rho_{i}}\left(\xi f_{\sigma}(\xi)\right)^{1 /(p-1)} d \xi,
\end{gathered}
$$

where the constant $C>0$ depends only on $n, p, \theta, \sigma, C_{1}$, and $C_{2}$. Hence,

$$
\begin{gathered}
\int_{M\left(\rho_{i+1}+0 ; u\right)}^{M\left(\rho_{i} ; u\right)}\left(g_{\theta}(t) t\right)^{-1 / p} d t+\int_{M\left(\rho_{i+1}+0 ; u\right)}^{M\left(\rho_{i} ; u\right)} g_{\theta}^{-1 /(p-1)}(t) d t \\
\quad \geq C \int_{\rho_{i+1}}^{\rho_{i}} \min \left\{\left(\xi f_{\sigma}(\xi)\right)^{1 /(p-1)}, q_{\sigma}^{1 / p}(\xi)\right\} d \xi
\end{gathered}
$$

for any $i \in\{0, \ldots, k-1\}$. Summing the last estimate over all $i \in\{0, \ldots, k-1\}$, we obtain

$$
\begin{gathered}
\int_{M\left(r_{1}+0 ; u\right)}^{M(r ; u)}\left(g_{\theta}(t) t\right)^{-1 / p} d t+\int_{M\left(r_{1}+0 ; u\right)}^{M(r ; u)} g_{\theta}^{-1 /(p-1)}(t) d t \\
\geq C \int_{r_{1}}^{r} \min \left\{\left(\xi f_{\sigma}(\xi)\right)^{1 /(p-1)}, q_{\sigma}^{1 / p}(\xi)\right\} d \xi
\end{gathered}
$$

On the other hand, condition (2.4) allows us to assert that

$$
\begin{aligned}
\int_{r_{1}}^{r} \min \left\{\left(\xi f_{\sigma}(\xi)\right)^{1 /(p-1)}, q_{\sigma}^{1 / p}(\xi)\right\} d \xi \geq & \frac{1}{2} \int_{r_{0}}^{r} \min \left\{\left(\xi f_{\sigma}(\xi)\right)^{1 /(p-1)}, q_{\sigma}^{1 / p}(\xi)\right\} d \xi \\
& +\frac{1}{C} \int_{M\left(r_{1}+0 ; u\right)}^{1}\left(g_{\theta}(t) t\right)^{-1 / p} d t \\
& +\frac{1}{C} \int_{M\left(r_{1}+0 ; u\right)}^{1} g_{\theta}^{-1 /(p-1)}(t) d t
\end{aligned}
$$

if the real number $r$ is large enough. Combining this with (3.30), we complete the proof.

Proof of Theorem 2.4. Let $u$ be a solution of (1.1), (1.2). If $M(\cdot ; u)$ is a bounded function on the interval $\left(r_{0}, \infty\right)$, then inequality (2.5) is obviously valid for all sufficiently large $r$ since, in this case, the right-hand side of (2.5) tends to zero as $r \rightarrow \infty$, whereas the left-hand side is bounded below by a positive real number for all $r \in\left(r_{0}, \infty\right)$. 
In turn, if $M(\cdot ; u)$ is not a bounded function, then (3.29) holds. We remind that $M(\cdot ; u)$ is a non-decreasing function on $\left(r_{0}, \infty\right)$ according to the maximum principle. Thus, estimate (2.5) follows immediately from Lemma 3.5.

The proof is completed.

Remark 3.2. From the arguments given above, it can be seen that Theorems 2.1 2.4 remain hold for solutions of the problem

$$
\left\{\begin{array}{l}
\operatorname{div} A(x, D u)+b(x)|D u|^{p-1} \geq q(x) g(u) \text { in } \Omega_{r_{0}, \infty}, \\
\left.u\right|_{\Gamma_{r_{0}, \infty}}=0,
\end{array}\right.
$$

where $\Omega_{r_{0}, \infty}=\left\{x \in \Omega: r_{0}<|x|\right\}$ and $\Gamma_{r_{0}, \infty}=\left\{x \in \partial \Omega: r_{0}<|x|\right\}$. In this case, in the hypotheses of Theorems 2.122 .3 , we must additionaly assume that $M(\cdot ; u)$ is a non-decreasing function on the interval $\left(r_{0}, \infty\right)$.

\section{REFERENCES}

[1] J.B. Keller, On solution of $\triangle u=f(u)$, Comm. Pure Appl. Math. 10 (4) (1957) 503-510.

[2] V.A. Kondratiev, E.M. Landis, Qualitative properties of the solutions of a second-order nonlinear equations, Mat. Sb. 135 (3) (1988) 346-360.

[3] A.A. Kon'kov, On properties of solutions of elliptic inequalities with a nonlinearity in the principal part, Doklady Mathematics 65 (2) (2002), 157-160.

[4] A.A. Kon'kov, On comparison theorems for elliptic inequalities, J. Math. Anal. Appl. 388 (2012), 102-124.

[5] O.A. Ladyzhenskaya, N.N. Ural'tseva, Linear and quasilinear elliptic equations, Academic Press, New York-London, 1968.

[6] E. Mitidieri, S.I. Pohozaev, Nonexistence of positive solutions for quasilinear elliptic problems on $\mathbb{R}^{n}$, Proc. V.A. Steklov Inst. Math. 227 (18) (1999) 192-222.

[7] E. Mitidieri, S.I. Pohozaev, A priori estimates and blow-up of solutions to nonlinear partial differential equations and inequalities, Proc. V.A. Steklov Inst. Math. 234 (2001).

[8] R. Osserman, On the inequality $\triangle u \geq f(u)$, Pacific J. Math. 7 (4) (1957), 1641-1647.

[9] L. Veron, Comportement asymptotique des solutions d'equations elliptiques semi-lineaires dans $\mathbb{R}^{n}$, Ann. Math. Pure Appl. 127 (1981) 25-50.

[10] L. Veron, Singularities of Solutions of Second Order Quasilinear Equations, Addison Wesley Longman Ltd., 1996.

Department of Differential Equations, Faculty of Mechanics and Mathematics, Moscow Lomonosov State University, Vorobyovy Gory, 119992 Moscow, Russia

E-mail address: konkov@mech.math.msu.su 